\title{
Fullerenes, Nanotubes, and Graphite as Matrices for Collision Mechanism in Secondary lon Mass Spectrometry: Determination of Cyclodextrin
}

\author{
Monika Stupavska, ${ }^{1}$ Monika Jerigova,,${ }^{1,2}$ Miroslav Michalka, ${ }^{2}$ Daniel Hasko, ${ }^{2}$ \\ Vojtech Szoecs, ${ }^{3}$ Dusan Velic ${ }^{1,2}$ \\ ${ }^{1}$ Department of Physical and Theoretical Chemistry, Faculty of Natural Sciences, Comenius University in Bratislava, Mlynska \\ dolina, 84215 Bratislava, Slovakia \\ ${ }^{2}$ International Laser Center, Ilkovicova 3, 84104 Bratislava, Slovakia \\ ${ }^{3}$ Institute of Chemistry, Faculty of Natural Sciences, Comenius University in Bratislava, Mlynska dolina, 84215 Bratislava, Slovakia
}

\begin{abstract}
A technique for improving the sensitivity of high mass molecular analysis is described. Three carbon species, fullerenes, single walled carbon nanotubes, and highly ordered pyrolytic graphite are introduced as matrices for the secondary ion mass spectrometry analysis of cyclodextrin $\left(\mathrm{C}_{42} \mathrm{H}_{70} \mathrm{O}_{35}, 1134 \mathrm{u}\right)$. The fullerene and nanotubes are deposited as single deposition, and 10,20 , or 30 deposition films and cyclodextrin is deposited on top. The cyclodextrin parent-like ions and two fragments were analyzed. A 30 deposition fullerene film enhanced the intensity of cationized cyclodextrin with $\mathrm{Na}$ by a factor of 37 . While the $\mathrm{C}_{6} \mathrm{H}_{11} \mathrm{O}_{5}$ fragment, corresponding to one glucopyranose unit, increased by a factor of 16 . Although fragmentation on fullerene is not suppressed, the intensity is twice as low as the parent-like ion. Deprotonated cyclodextrin increases by $100 x$ and its $\mathrm{C}_{8} \mathrm{H}_{7} \mathrm{O}$ fragment by $10 x$. While the fullerene matrix enhances secondary ion emission, the nanotubes matrix film generates a basically constant yield. Graphite gives rise to lower intensity peaks than either fullerene or nanotubes. Scanning electron microscopy and atomic force microscopy provide images of the fullerene and nanotubes deposition films revealing flat and web structured surfaces, respectively. A "colliding ball" model is presented to provide a plausible physical mechanism of parentlike ion enhancement using the fullerene matrix.
\end{abstract}

Key words: ToF-SIMS, Matrix effect, Fullerene, Nanotube, Graphite, Yield enhancement

\section{Introduction}

$\mathrm{M}$ ass Spectrometry (MS) investigation of large organic molecules has received enormous and increasing attention. The majority of these studies are focused on the

Electronic supplementary material The online version of this article (doi:10.1007/s13361-011-0239-0) contains supplementary material, which is available to authorized users.

Correspondence to: Monika Stupavska; e-mail: monika.stupavska@gmail. com improvement of laser desorption ionization methods, of which the most successful is matrix assisted laser desorption ionization (MALDI). Siuzdak et al. introduced an efficient matrix-free method termed Desorption Ionization on Silicon (DIOS) [1, 2]. The performance of their nanoporous silicon surfaces was evaluated on a wide range of analytes. The dependences between nanostructured surfaces and secondary ion (SI) emission were later demonstrated for various types of nanomaterials $[3,4]$.

In the case of secondary ion mass spectrometry (SIMS), a laser free method, sample preparation techniques are mainly 
used to improve SI emission. The most common of these is matrix-enhanced SIMS (ME-SIMS). This technique combines SIMS with a sample preparation similar to that used for MALDI [5-9]. Alkali and transition metal salts have been already used as an alternative of the low molecular weight matrices [10]. The other technique that has been developed for improving sensitivity is sample metallization metal assisted SIMS (MetA-SIMS). This technique builds on beneficial effects exhibited by layers of gold $[11,12]$ and silver clusters [13].

The first use of fullerenes and single-walled carbon nanotubes (SWCNT) in MS was as matrices for MALDI $[4,14,15]$. In recent years, advances in the chemistry of functionalized fullerenes and SWCNT have resulted in many applications and these derivatized fullerenes and SWCNT have been shown to be efficient matrices for MALDI mass spectrometry [16-19].

Pure nanostructured carbon matrices have not yet been tested for SIMS analysis. Here, we introduce nanostructured carbon species as matrices for the SIMS analysis of a $\beta$ cyclodextrin (CD) large organic molecule. In order to investigate and understand the matrix effect, we focused on the emission processes of an intact $\mathrm{CD}$ analyte and its fragmentation during SIMS analysis. The coverage or surface concentration of $\mathrm{CD}$ was kept constant in order to study the matrix effect and not the effect of analyte layer thickness. The characteristic CD-parent-like ions are determined from SIMS spectra and analyzed as a function of increasing number of carbon species depositions forming the matrix film. The SI yield of parent-like ions was then correlated not only with different carbon species and their depositions, but also with the morphology and topography of the matrix film surface. The matrix film surface was visualized by scanning electron microscopy (SEM) and atomic force microscopy (AFM). The surface properties of these $\mathrm{CD} /$ carbon matrix systems are examined in order to obtain detailed information about the carbon matrices, which participate in SI generation either in the form of parent-like ions or as fragments.

\section{Experimental}

\section{Matrix Preparation}

Three carbon matrices were used: fullerene (Sigma Aldrich (Bratislava, Slovakia), 99. 5\% purity), single-walled carbon nanotubes (SWCNT; Sigma Aldrich, 90\%), and highly ordered pyrolytic graphite (HOPG; SPI Supplies (West Chester, PA), $10 \times 10 \times 2 \mathrm{~mm}$ ). Fullerene and SWCNT matrix carbon species were prepared from solution and HOPG surface was freshly prepared before each experiment by cleaving with adhesive tape. The fullerene and SWCNT species were dissolved and diluted in toluene and were prepared as thin films by spin coating $20 \mu \mathrm{L}$ of solution on a $1 \mathrm{~cm}^{2} \mathrm{Si}$ wafer. The Si wafer was precleaned by hydrofluoric acid, ultrasonication in acetone, and air drying prior to deposition. The fullerene matrix solution was prepared by dissolving $3.2 \mathrm{mg}$ of $\mathrm{C}_{60}$ in $1 \mathrm{ml}$ of toluene. This gave a coverage of $10^{16}$ fullerene molecules per $1 \mathrm{~cm}^{2}$. The SWCNT matrix solution was prepared by dissolving $0.1 \mathrm{~g}$ of SWCNT in $10 \mathrm{~mL}$ of toluene, when this solution was deposited on $1 \mathrm{~cm}^{2}$ of $\mathrm{Si}$ wafer. The surface coverage is approximately $10^{14}$ SWCNT molecules per $1 \mathrm{~cm}^{2}$. The surface coverage was estimated based on the sizes of the fullerene and SWCNT molecules lacking specific surface binding sites. The diameters of a fullerene ball and SWCNT are approximately $1 \mathrm{~nm}$, while the length of a nanotube is approximately $1000 \mathrm{~nm}$. Since the geometries of fullerene and SWCNT are different, the matrix surface coverage was based on the number of $\mathrm{C}$ atoms involved in the matrix. The calculated surface coverages, for fullerene and SWCNT matrices, were $10^{16}$ and $10^{14}$ molecules $/ \mathrm{cm}^{2}$, respectively, giving a normalized $\mathrm{C}$ atom coverage of approximately $10^{18} \mathrm{C}$ atoms per $1 \mathrm{~cm}^{2}$ of matrix in both cases. Note that the concentrations of the deposited solutions and the surface area of the $\mathrm{Si}$ wafer were purposely chosen in order to have a constant number of $\mathrm{C}$ atoms. Care was taken to prepare each matrix in the same way, and the surface coverage of a single deposition does correspond well to the monolayer (one deposition corresponds to $20 \mu \mathrm{L}$ drop). Nevertheless, the preparation process is defined by the number of depositions and not by the number of monolayers. Moreover, that multiple deposition appears to be quite complex.

\section{Analyte Preparation}

$\beta$-Cyclodextrin ( $\mathrm{CD}$, Cyclolab) with a molecular weight of $1134 \mathrm{u}$ was used as a model analyte for SIMS measurements. A $20 \mu \mathrm{L}$ drop of a $0.01 \mathrm{M}$ aqueous solution of $\mathrm{CD}$ was spin coated to form the uppermost thin layer on the carbon type matrix films supported on the $\mathrm{Si}$ wafer. Only one $\mathrm{CD}$ deposition was performed, so the coverage or surface concentration of the CD layer was always the same. Note that the overall preparation of the $\mathrm{CD} /$ carbon matrix film system was performed in two steps, first the carbon species deposition and carbon species film formation, and second the CD deposition of this thin carbon species film. This approach is obviously different than that used in MALDI, since a SIMS technique is based on high surface sensitivity. The samples were dried out on air at room temperature.

\section{Time of Flight Secondary Ion Mass Spectrometry}

These experiments were carried out with a SIMS instrument (TOF-SIMS IV, ION-TOF) reflectron type time-of-flight (TOF) mass spectrometer equipped with a bismuth ion gun. Pulsed $25 \mathrm{keV} \mathrm{Bi}_{3}{ }^{+}$were used as primary ions with an ion current of $0.25 \mathrm{pA}$. SIMS spectra were measured by scanning over a selected $100 \times 100 \mu \mathrm{m}^{2}$ analysis area with a total primary ion dose density below the static limit of $5 \times 10^{12}$ ions $/ \mathrm{cm}^{2}$. To prevent any charging of the surface, an electron flood gun was used. 


\section{Scanning Electron Microscopy}

The morphology and topography of the sample surfaces were characterized using scanning electron microscopy (SEM, LEO 1550). The imaged area was approximately $100 \times 100 \mu \mathrm{m}^{2}$, where the images were formed mostly by secondary and backscattered electrons, providing the topography and the contrast, respectively.

\section{Atomic Force Microscopy}

Measurements of surface morphology, topography, and roughness were carried out using an air conditioned atomic force microscopy (AFM, NT-MDT). The measurements were performed in semicontact mode using a tip with a radius of $10 \mathrm{~nm}$ and a selected area of $2 \times 2 \mu \mathrm{m}^{2}$.

\section{Results and Discussion}

\section{Fullerene Matrices}

Positive SIMS spectra of CD on fullerene matrix are shown in Figure 1, parent-like ions and representative fragments are emphasized. The results show the yields of CD parent-like ions from the matrices with different numbers of fullerene depositions. The SIMS spectra are dominated by the $\mathrm{CD}\left(\mathrm{C}_{42} \mathrm{H}_{70} \mathrm{O}_{35}\right.$, $1134 \mathrm{u}$ ) parent-like ions, which are observed in cation forms with bound $\mathrm{Na}$ and $\mathrm{K}$ at 1157 and $1173 \mathrm{u}$, respectively. The intensities of the parent-like ions as $\mathrm{CD}+\mathrm{Na}$ and $\mathrm{CD}+\mathrm{K}$ from the single fullerene deposition film are $621 \mathrm{cts} / 0.93$ and $557 \mathrm{cts} /$ 0.74 , respectively. The average integral intensity values and their respective standard deviations are based on six measurements, and the normalized intensity values are relative to the total ion counts. Increasing the number of fullerene depositions resulted in surfaces with higher fullerene coverage or thicker films of fullerene matrix. The $\mathrm{CD}+\mathrm{Na}$ peak with the highest intensity is that of the parent-like ion and therefore the intensity of this peak was used for a quantitative comparison based on normalized intensities to total ion counts. The intensities of CD $\mathrm{SI}$ are summarized in Table 1 . The SI yield of $\mathrm{CD}+\mathrm{Na}$ from a fullerene matrix consisting of 10 depositions is approximately eight times higher than the yield from a single fullerene film reference sample (Figure 1b). For the 20 deposition fullerene matrix, the intensity of the $\mathrm{CD}$ parent-like ion increased approximately 31 times in comparison to a single fullerene film reference sample (Figure 1c). For the 30 deposition fullerene matrix, the effect of enhancement becomes even stronger: a 30 deposition fullerene matrix film enhanced the SI intensity of $\mathrm{CD}+\mathrm{Na}$ by a factor of approximately 37 (Figure 1d).

The fullerene matrices influenced not only the CD parentlike ion emission, but also the fragmentation of $\mathrm{CD}$. Fragments weighting 163 and $325 \mathrm{u}$, which correspond to one $\left(\mathrm{C}_{6} \mathrm{H}_{11} \mathrm{O}_{5}\right)$ and two $\left(\mathrm{C}_{12} \mathrm{H}_{21} \mathrm{O}_{10}\right)$ glucopyranose units, respectively, were analyzed. The data show that a 30 deposition fullerene matrix does not decrease the fragmentation of $\mathrm{CD}$ compared with a single deposition fullerene
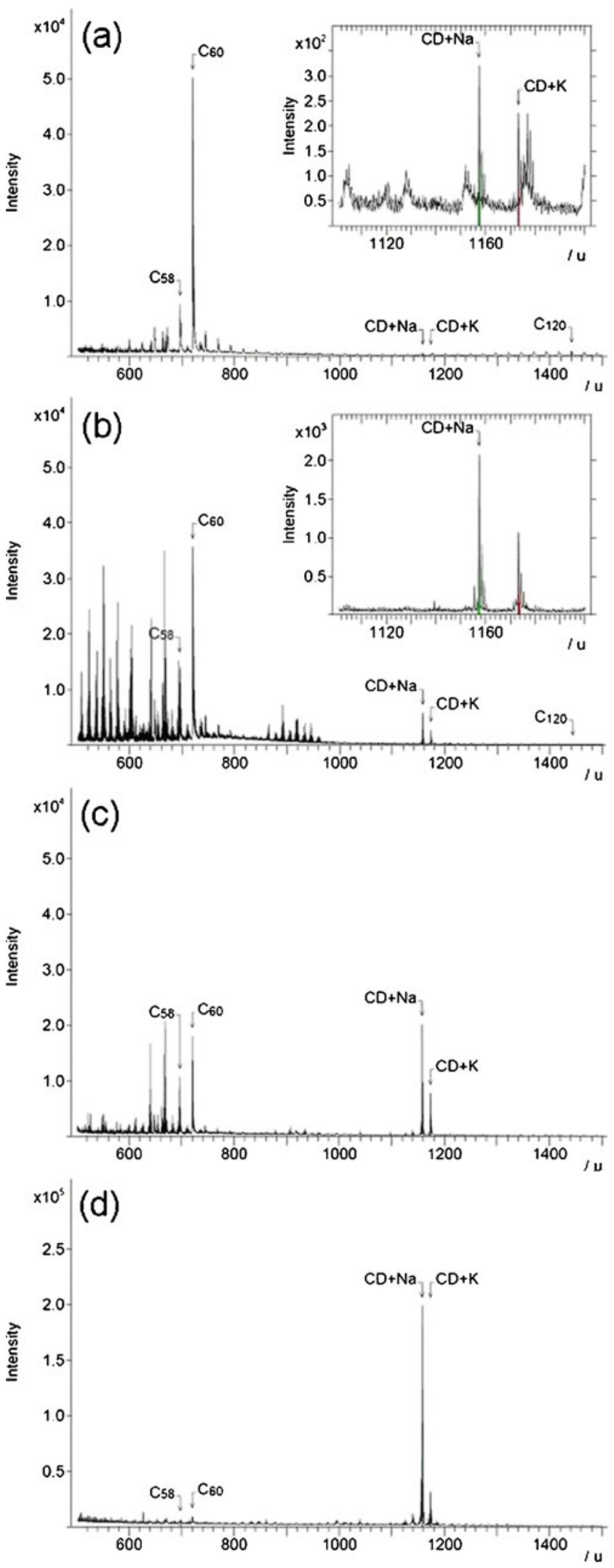

Figure 1. Positive SIMS spectra of CD on fullerene matrices, (a) 1 deposition, (b) 10 depositions, (c) 20 depositions, and (d) 30 depositions 
Table 1. Emission Yields of CD Cations Obtained from Fullerene, SWCNT, and HOPG Matrices

\begin{tabular}{|c|c|c|c|c|c|c|c|}
\hline \multirow{4}{*}{ Matrix } & \multirow{4}{*}{$\begin{array}{l}\text { Number of } \\
\text { depositions }\end{array}$} & \multicolumn{6}{|c|}{ Positive ions } \\
\hline & & \multirow{2}{*}{\multicolumn{2}{|c|}{$\begin{array}{c}\text { Parent-like ion } \\
\mathrm{CD}+\mathrm{Na}(1157 \mathrm{u})\end{array}$}} & \multicolumn{4}{|c|}{ Fragments } \\
\hline & & & & \multicolumn{2}{|c|}{$\mathrm{C}_{6} \mathrm{H}_{11} \mathrm{O}_{5}(163 \mathrm{u})$} & \multicolumn{2}{|c|}{$\mathrm{C}_{12} \mathrm{H}_{21} \mathrm{O}_{10}(325 \mathrm{u})$} \\
\hline & & $\mathrm{I}_{n}$ & $\mathrm{I}_{i n t}$ & $\mathrm{I}_{n}$ & $\mathrm{I}_{i n t}$ & $\mathrm{I}_{n}$ & $\mathrm{I}_{i n t}$ \\
\hline $\mathrm{C}_{60}$ & 1 & 0.93 & $621 \pm 98$ & 18.29 & $12404 \pm 2168$ & 7.41 & $5218 \pm 462$ \\
\hline $\mathrm{C}_{60}$ & 10 & 7.83 & $7392 \pm 766$ & 143.57 & $161251 \pm 16255$ & 73.18 & $63436 \pm 4562$ \\
\hline $\mathrm{C}_{60}$ & 20 & 28.24 & $31577 \pm 2175$ & 179.36 & $207355 \pm 34399$ & 76.54 & $97287 \pm 36543$ \\
\hline $\mathrm{C}_{60}$ & 30 & 34.50 & $218785 \pm 7225$ & 296.51 & $1927565 \pm 150578$ & 124.20 & $771474 \pm 17854$ \\
\hline SWCNT & 1 & 104.82 & $141526 \pm 17076$ & 674.44 & $1204512 \pm 120006$ & 400.77 & $635377 \pm 56778$ \\
\hline SWCNT & 10 & 60.19 & $82792 \pm 10014$ & 596.04 & $1068229 \pm 71461$ & 283.32 & $470095 \pm 64298$ \\
\hline SWCNT & 20 & 193.27 & $374418 \pm 50684$ & 639.45 & $1185631 \pm 117782$ & 353.65 & $573185 \pm 82650$ \\
\hline SWCNT & 30 & 181.36 & $362517 \pm 37426$ & 507.64 & $1061481 \pm 42405$ & 231.70 & $466663 \pm 3510$ \\
\hline HOPG & - & 10.12 & $4262 \pm 469$ & 53.08 & $16507 \pm 6388$ & 21.28 & $10086 \pm 1620$ \\
\hline
\end{tabular}

$\mathrm{I}_{n}=$ normalized intensity; $\mathrm{I}_{\text {int }}=$ integral intensity (cts).

matrix. Although the fragmentation of the $\mathrm{CD}$ molecule on the fullerene matrices was not suppressed, the fragmentation is approximately twice as low as emission of the parent-like ion. These results indicate that the increased yields could reasonably arise as a consequence of a collision between the primary ions and the fullerene matrix. A uniform fullerene matrix system might therefore promote efficient collisions, leading to more efficient emission of intact molecules due to the "colliding ball" effect. This mechanism allows energy to be transferred through the centrosymetric fullerene ball into the whole system, maximizing the impact on the surface to sufficiently stimulate the emission of the parent-like ions in larger amounts. This trend is seen not only in the results where the CD molecular ion yield increases with increasing number of fullerene depositions, but also by the appearance of a CD dimer. The molecular ion corresponding to a CD dimer at $2292 \mathrm{u}$ is observed for the 30 deposition fullerene matrix but is not observed at all in the spectra from the single fullerene deposition.

In the mass region between 100 and $1500 \mathrm{u}$, molecular ions and fragments coming from fullerenes were identified. In the positive SIMS spectra shown in Figure 1a, it can be seen that the fragmentation of the fullerene matrix is more extensive for single fullerene deposition than for higher depositions. The collision energy is presumably redistributed within the thin fullerene-Si wafer interface, resulting in fullerene fragmentation. There is then an assumption that a large portion of the collision cascade energy is consumed by the fragmentation of the fullerenes and that only a small portion remains available to effectively eject the intact CD molecules. The fullerene fragments might additionally break the bonds of the CD molecule leaving only a small amount of the CD analyte as intact molecules in the sputtering process. Increasing the number of fullerene depositions would then suppress both fullerene fragmentation and the fullerene parent-like ion. The primary ion kinetic energy is evenly dissipated during collision cascade into the fullerene film and, in the 30 fullerene deposition matrix film; fragmentation of the fullerene makes only minor contributions to the sputter yield (Figure 1d).

To confirm the observations from the positive SIMS spectra, negative SIMS spectra were also determined (Supplementary Information S1). The major peak of interest is the deprotonated ion CD-H at $1133 \mathrm{u}$. In comparison with the single fullerene matrix film, the interaction of primary ions with a 30 deposition fullerene matrix film results in the emission of a much larger quantity of parent-like CD ions. For the 30 deposition fullerene matrix, the intensity of the CD-H parent-like ion increased approximately 100 times in comparison with a single deposition fullerene film reference sample. The fullerene matrices also influenced the fragmentation of CD. The intensity of the $119 \mathrm{u}$ fragment of $\mathrm{C}_{8} \mathrm{H}_{7} \mathrm{O}$, which represents one glucopyranose unit of the $\mathrm{CD}$ molecule was enhanced by a factor of approximately 10 . The results are summarized in Table 2. The intensity enhancement ratios in positive and negative SIMS measurements are clearly different due to the different ionization, fragmentation, and sputtering processes, however the increasing trend was maintained. These observations suggest that a fullerenebased modification of the analyte environment could be successfully applied to the surface characterization of complex organic systems.

To understand the SI enhancement observed in SIMS experiments based on matrix effects, a series of surface geometry investigations were performed, because the structural features of the surface are crucial for understanding and controlling the emission process of SIMS. The geometry of the fullerene depositions forming the matrix film was determined using SEM and AFM. The SEM images are shown in Figure 2a-d. A cleaned Si wafer was used as the substrate for all measurements. The images in Figure $2 \mathrm{a}-\mathrm{d}$ show the surface of the fullerene matrix after 1, 10, 20, and 30 depositions of fullerene solution, respectively. These depositions formed a flat surface with some scattered particles of clustered fullerene molecules as a result of local spots with higher fullerene concentration formed during the 
Table 2. Emission Yields of CD Anions Obtained from Fullerene, SWCNT, and HOPG Matrices

\begin{tabular}{|c|c|c|c|c|c|}
\hline \multirow{4}{*}{ Matrix } & \multirow{4}{*}{$\begin{array}{l}\text { Number of } \\
\text { depositions }\end{array}$} & \multicolumn{4}{|c|}{ Negative ions } \\
\hline & & \multicolumn{2}{|c|}{ Parent-like ion } & \multicolumn{2}{|c|}{ Fragment } \\
\hline & & \multicolumn{2}{|c|}{ CD-H (1133 u) } & \multicolumn{2}{|c|}{$\mathrm{C}_{8} \mathrm{H}_{7} \mathrm{O}(119 \mathrm{u})$} \\
\hline & & $\mathrm{I}_{n}$ & $\mathrm{I}_{i n t}$ & $\mathrm{I}_{n}$ & $\mathrm{I}_{i n t}$ \\
\hline $\mathrm{C}_{60}$ & 1 & 1.62 & $393 \pm 23$ & 94.72 & $23716 \pm 2365$ \\
\hline $\mathrm{C}_{60}$ & 30 & 162.57 & $89795 \pm 2965$ & 995.35 & $805242 \pm 129843$ \\
\hline SWCNT & 1 & 97.56 & $133463 \pm 16848$ & 291.62 & $492367 \pm 88652$ \\
\hline SWCNT & 30 & 69.36 & $57508 \pm 630$ & 641.40 & $915688 \pm 132972$ \\
\hline HOPG & - & 0.79 & $145 \pm 24$ & 15.23 & $2445 \pm 580$ \\
\hline
\end{tabular}

$\mathrm{I}_{n}=$ normalized intensity; $\mathrm{I}_{\text {int }}=$ integral intensity (cts)

spin coating deposition. The AFM images were also used to determine the morphology and topography or roughness of the fullerene matrix surface. Figure $2 \mathrm{e}$ shows a semicontact AFM scan of a 30 deposition fullerene film. The surface is flat with an average roughness of $0.95 \mathrm{~nm}$. The fullerene clusters are also observed in the AFM image. It is important to stress that all SIMS measurements were performed on carefully selected flat areas, which were free of the observed clusters of fullerene since this investigation focused on the role of a flat fullerene matrix.

\section{Carbon Nanotubes Matrices}

SWCNT were also used as a matrix for the characterization of CD molecules using SIMS. Analysis of CD on the SWCNT matrix is based again on an increasing number of SWCNT depositions. The overall increase of the $\mathrm{CD}+\mathrm{Na}$ parent-like ion peak is much less significant compared with the enhancement seen in the fullerene matrix (Supplementary Information S2). The results of the $\mathrm{CD}+\mathrm{Na}$ parent-like ion intensities are compared and summarized in Table 1, showing that there are only slight differences in SI yield as the number of SWCNT depositions increases. The fluctuation of the intensity factors within a factor of two suggests that the increasing surface coverage of SWCNT has no significant effect on the intensity of the CD parent-like ion in the SIMS measurements. Interestingly, SWCNT matrices generate very low intensity carbon fragments in the SIMS spectra, which can be easily separated from the $\mathrm{CD}$ analyte related peaks.

The SWCNT matrices might influence not only the parent-like ion emission, but also the fragmentation of $\mathrm{CD}$. The SI yield of the 163 and $325 \mathrm{u}$ fragments is shown in Table 1. It appears that the peak intensities of these CD fragments even slightly decrease with higher depositions.

The negative SIMS spectra were also determined (Supplementary Information S3) where the deprotonated CD-H parent-like ion weights 1133 u. For the 30 deposition SWCNT matrix, the intensity of the $\mathrm{CD}-\mathrm{H}$ parent-like ion decreased approximately 1.5 times in comparison with a single SWCNT film reference sample. The SWCNT matrix also influenced fragmentation. The SWCNT matrices provided an enhanced peak from the $\mathrm{C}_{8} \mathrm{H}_{7} \mathrm{O}$ fragment. The enhancement is a factor of approximately two. The results are summarized in Table 2.

The source of the behavior exhibited by the SWCNT is clearly different from that of the fullerene matrices.
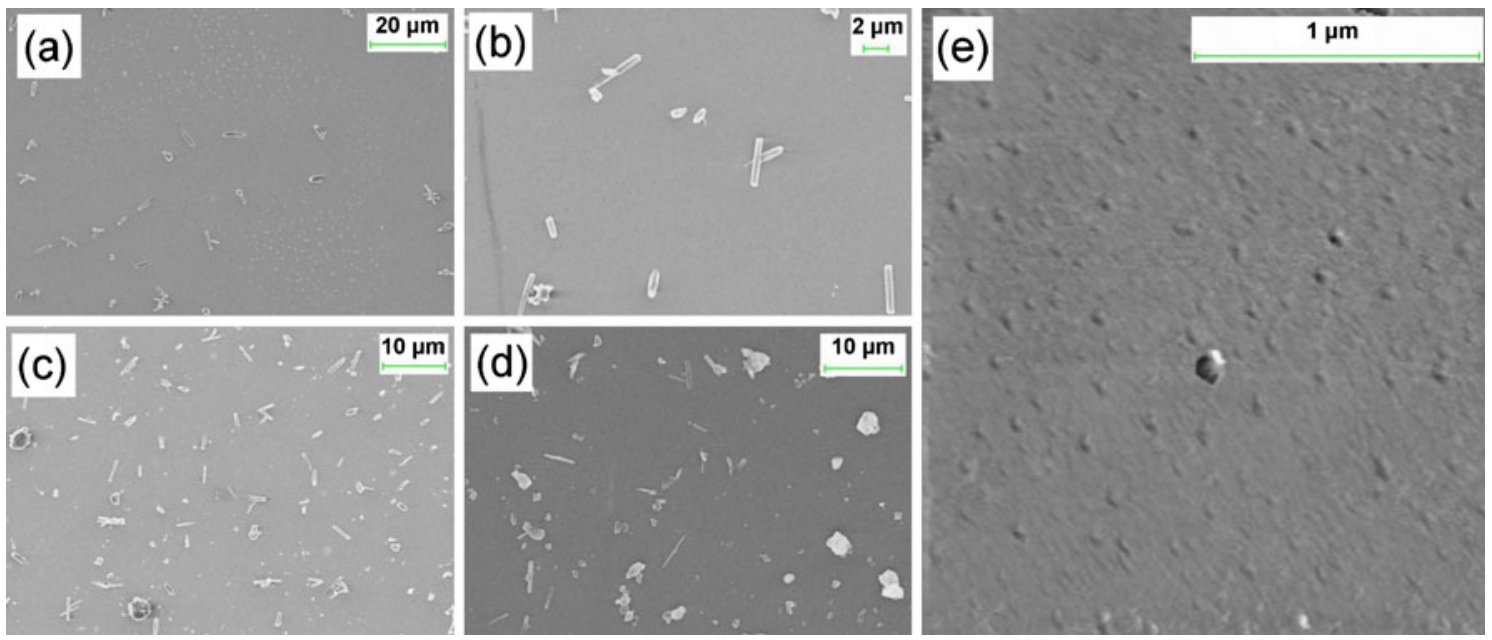

Figure 2. SEM images of fullerene matrices, (a) 1 deposition, (b) 10 depositions, (c) 20 depositions, (d) 30 depositions on Si, (e) AFM image of 30 depositions of $\mathrm{C}_{60}$ on $\mathrm{Si}$ 
Although a great deal of effort was exerted to prepare relatively flat SWCNT films, it is still difficult to control its surface deposition. SEM images shown in Figure 3 show that the SWCNT matrix films consist of entangled nanotubes forming a web structure. The images in Figures $3 \mathrm{a}-\mathrm{d}$ reveal the SWCNT web morphology, where aggregation of SWCNT due to their large van der Waals attractions not only prevents formation of the intended films, but also causes some heterogeneities. These heterogeneities include defects or pinholes in the SWCNT matrix surface, which might cause repression of the analyte signals. The SWCNT deposition was also examined using AFM measurements as shown in Figure 3e. This AFM image of a 30 SWCNT deposition surface supports the SEM images revealing the web structure with a roughness of $22.41 \mathrm{~nm}$. This SWCNT roughness is approximately 20 times higher than the roughness of the fullerene.

\section{Highly Ordered Pyrolytic Graphite Matrix}

The efficiency of CD parent-like ion emission was determined on two carbon based species, fullerene and SWCNT, with significantly different results. While the fullerene matrix enhanced the CD parent-like ion emission in SIMS analysis as a function of increasing fullerene surface coverage, the SWCNT matrix generated a basically constant SI yield as a function of different SWCNT surface coverage. A logical choice for a third carbon species is graphite, in particular HOPG. Small, nonspecific organic fragments from the HOPG matrix and the CD analyte dominate the low mass region of the SIMS spectra (Supplementary Information S4). The respective peaks have lower intensities than those seen in the cases of fullerene and SWCNT. Since SIMS analysis is limited to the first few monolayers of the sample, the highest SI yield events occur when enough energy from the primary ions is dissipated within the top few monolayers of the sample surface [20]. Molecular dynamics simulations show that the graphite is a very open substrate; therefore the primary ion can penetrate into the sample bulk, leaving little energy in the surface region to promote sputtering [21]. The same behavior is observed for $\mathrm{CD}$ fragmentation. For the 163 and $325 \mathrm{u}$ ions, yields are lower than from the fullerene matrix. It seems that the emission of these fragments is repressed. The same results were determined in negative polarity. The peaks are assigned as a set of carbon clusters $\mathrm{C}_{\mathrm{n}}{ }^{-}$and $\mathrm{CD}-\mathrm{H}$ parent-like ion with low intensity. HOPG creates a morphologically and topographically flat surface without any defects and with minimal roughness, as expected and confirmed using SEM and AFM (images not shown).

\section{Comparison of Carbon Matrices}

The SEM images of carbon species matrices overlayered with CD layer are shown in Figure 4. Figure 4a shows the $\mathrm{CD} /$ fullerene system revealing an even distribution of $\mathrm{CD}$ molecules with some surface features probably due to the fullerene film structure. The overall structure of this $\mathrm{CD} /$ fullerene system seems to be flat. The SWCNT matrix with a $\mathrm{CD}$ layer is shown in Figure 4b. The web morphology is masked by the analyte molecules adsorbed on the surface of the carbon nanotube layers. It can be seen that the SWCNT layers can even act as a medium for the crystallization of $\mathrm{CD}$, which is not observed in the fullerene matrix. The flat surface in Figure 4c shows the HOPG matrix with a CD layer. Based on these surface morphologies and the SIMS results, a "colliding ball" mechanism might be proposed to account for the enhancement of SI emission. The key features of this model are shown in Figure 5. The film of fullerene molecules with $\mathrm{CD}$ and the collision penetration into this layer are illustrated in Figure 5a and b, respectively. In order to sputter the large CD molecule with minimal fragmentation, the SIMS mechanism relies on recoil collisions from the bulk towards the surface. This mechanism
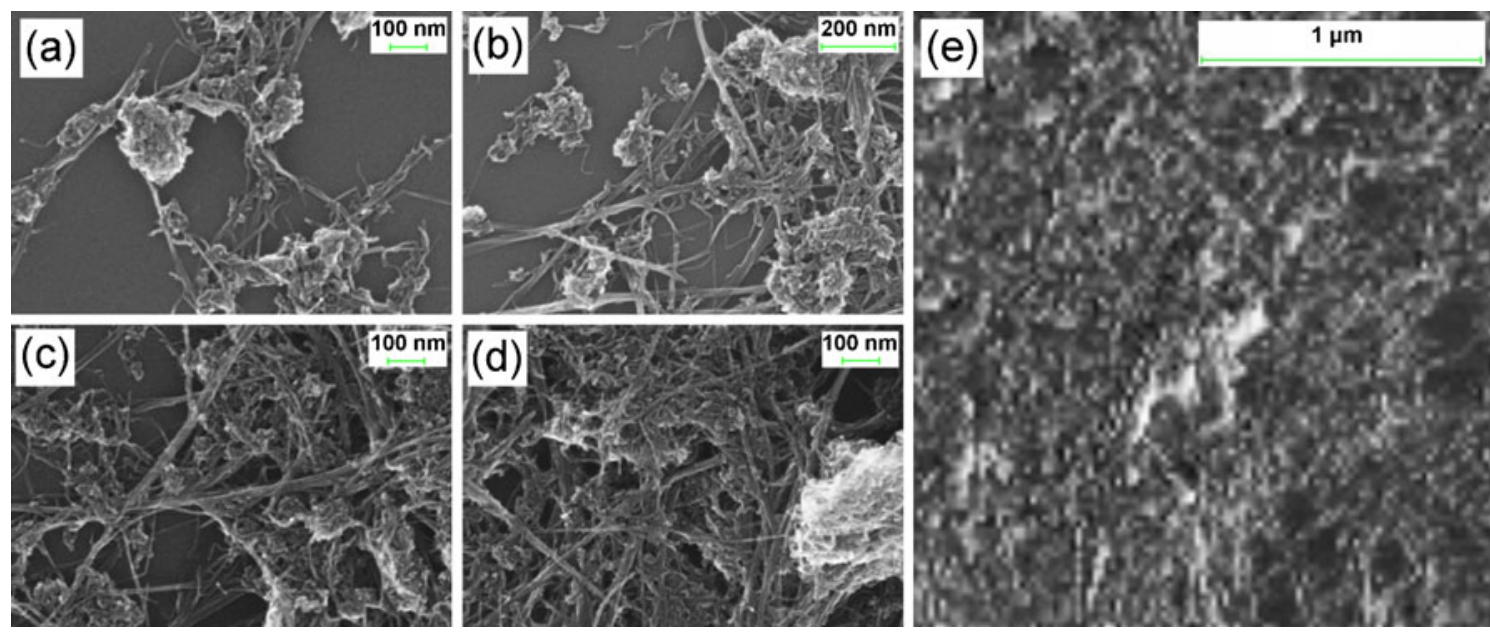

Figure 3. SEM images of SWCNT matrices, (a) 1 deposition, (b) 10 depositions, (c) 20 depositions, (d) 30 depositions on Si, (e) AFM image of 30 depositions of SWCNT on $\mathrm{Si}$ 

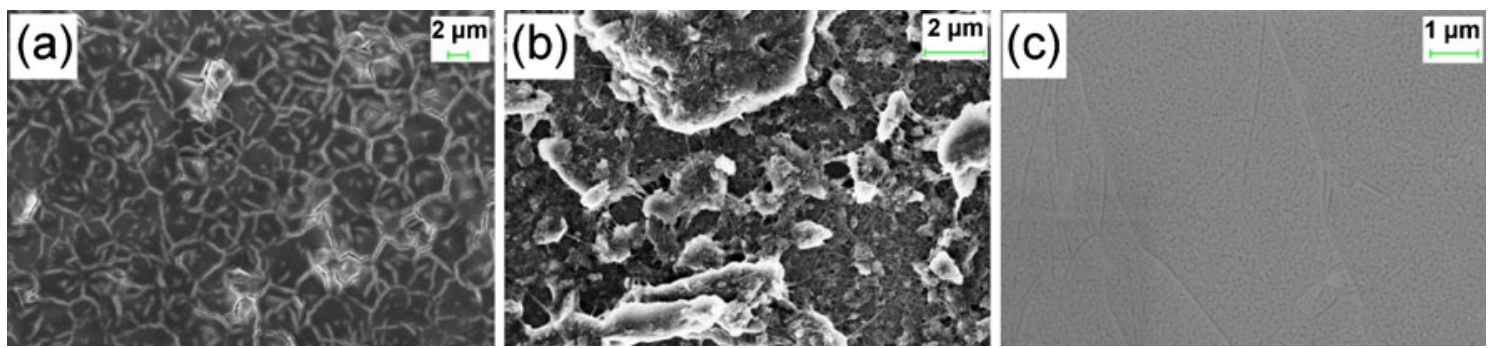

Figure 4. SEM images of morphology of CD analyte deposited onto (a) fullerene matrix, (b) SWCNT matrix, (c) HOPG matrix

allows energy to be transferred through the fullerene ball centrosymmetric system into the whole system evenly in all directions. In such a way, a large portion of the energy is transferred to the surface, maximizing the effect of the primary ion impact and stimulating sputtering in larger amounts. Note that the collision of the primary Bi ion is not discussed in this illustration, since the primary impact atomizes all molecules at the site of impact and the parentlike ions are coming from the side of the impact zone due to recoil collisions. The recoil collision transfers energy from the bulk $\mathrm{Si}$ into the matrix, and such a collision travels along the fullerene $\mathrm{C}-\mathrm{C}$ bonds. A classic model of collision is able to describe energy transfer correctly from an impacting $\mathrm{Si}$ projectile to a $\mathrm{C}_{60}$ ball [22]. The projectile molecule must have energy above a certain threshold in order for mechanical effects to occur rather than electronic scattering $[23,24]$. The model requires that the $\mathrm{Si}-\mathrm{C}$ interaction time is shorter than the $\mathrm{C}$-fullerene oscillation period, i.e., the interacting $\mathrm{C}$-fullerene is relatively static and the rest of the $\mathrm{C}_{60}$ acts as a "spectator." The consequent energy transfer occurs through high-energy vibrations of $\mathrm{C}_{60}$, corresponding to intermolecular $\mathrm{C}-\mathrm{C}$ interactions, with energies of around $1480 \mathrm{~cm}^{-1}$, which are predominantly tangential to the $\mathrm{C}_{60}$ sphere [23, 24]. Figure 5b illustrates the most probable impact of $\mathrm{Si}$, radial to the $\mathrm{C}_{60}$ sphere. The initial impulse $\mathrm{p}_{0}$ is decomposed into tangential local vibrations following the nearest $\mathrm{C}-\mathrm{C}$ interactions. The global direction of energy transfer corresponds to the rotation axis of $\mathrm{C}_{60}$. Next, in accordance with the $\mathrm{C}_{60}$ geometry, each vibration evolves subsequent vibrations of the nearest fullerene molecules. Sequential waves of these vibration steps are responsible for energy transfer from the side of $\mathrm{C}_{60}$ adjacent to the $\mathrm{Si}$ to the opposite side, preserving almost full efficiency with the final impulse $p_{1}$. Note that in the case of fullerene, the final (a)

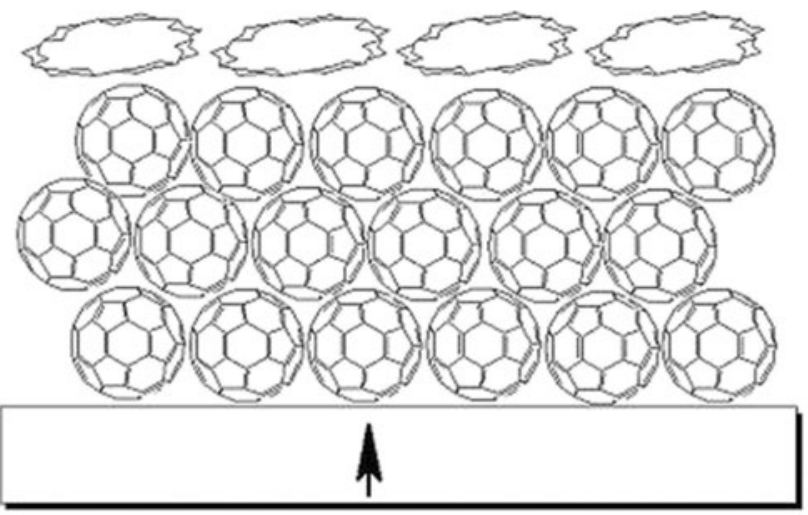

(c)
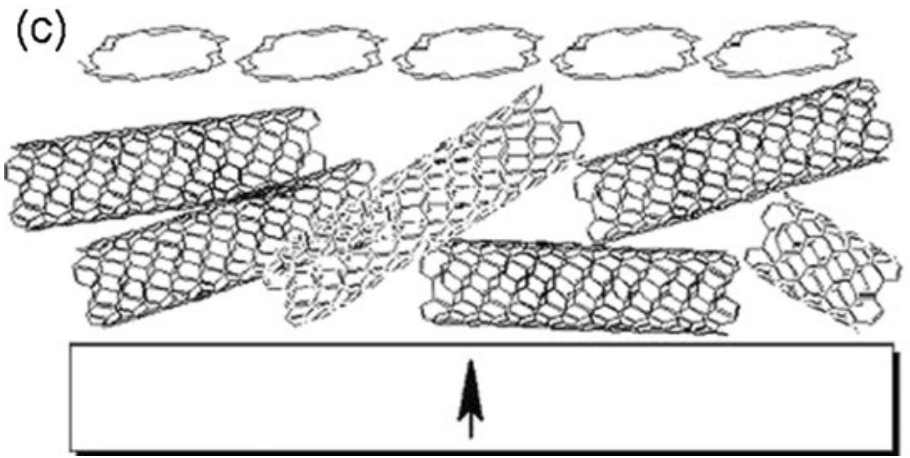

(b)

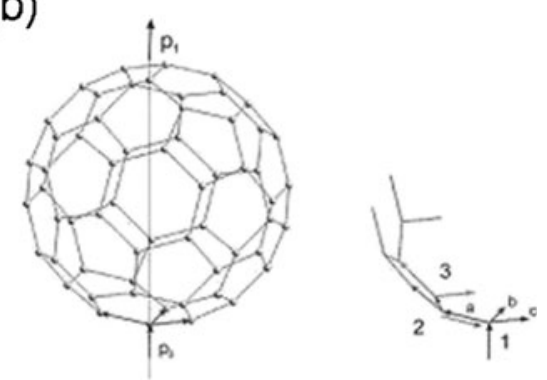

(d)

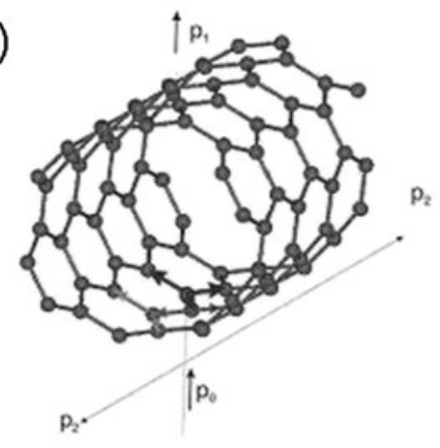

Figure 5. Scheme of a "colliding ball" mechanism and general view of geometry after impact of Si (impulse $\mathrm{p}_{0}$ ) and impulse decomposition (impulses $\mathrm{p}_{1}$ and $\mathrm{p}_{2}$ ), (a), (b) fullerene matrix, (c), (d) SWCNT matrix 
impulse $\mathrm{p}_{1}$ is also located at the top of the fullerene molecule.

In contrast, the energy transfer within the carbon nanotube is less effective. The film of SWCNT with CD and its collision penetration are illustrated in Figure $5 \mathrm{c}$ and $\mathrm{d}$, respectively. The recoil collision travels from the $\mathrm{Si}$ atom into the SWCNT molecule. Note that the initial impulse $\mathrm{p}_{0}$ is decomposed into at least two directions. One direction is along the diameter of the SWCNT tube, congruent with the final impulse $\mathrm{p}_{1}$. The second direction is along the length of the SWCNT tube resulting in another final impulse $\mathrm{p}_{2}$. Assuming the web structure along the surface, the final impact $\mathrm{p}_{2}$ has no impact on the sputtering of the CD molecule in the vertical direction towards the SIMS analyzer. The final impulse $p_{1}$, which is responsible for the sputtering of CD in the SIMS analysis, has approximately half of the fullerene matrix efficiency. In conclusion, the increase in $\mathrm{CD}$ ion yield appears to be the result of a change in the collision mechanism due to the geometry of the matrix, rather than to chemical modification of the matrix, because only carbon species are used. Nevertheless, the effect of temperature on CD SI yield on the fullerene matrix is currently being investigated in order to address the role of possible fullerene sublimation.

\section{Conclusions}

Three carbon species, fullerenes, SWCNT, and HOPG, were introduced as matrices for the SIMS analysis of cyclodextrin. Single and 10, 20, and finally 30 depositions were used to prepare the matrix films. The $\mathrm{CD}\left(\mathrm{C}_{42} \mathrm{H}_{70} \mathrm{O}_{35}, 1134\right.$ u) parent-like ions were observed in cationized forms with $\mathrm{Na}$ and $\mathrm{K}$ at 1157 and $1173 \mathrm{u}$, respectively. The 30 deposition fullerene matrix enhanced the SI intensity of $\mathrm{CD}+\mathrm{Na}$ by factor of approximately 37 . Although fragmentation of $\mathrm{CD}$ on the fullerene matrices was not suppressed, the fragmentation is approximately twice as low as parent-like ion emission. SI enhancement was observed for both polarities, suggesting that primary energy transfer and efficient sputtering is favored by fullerene matrix films, which might act as an energy transfer medium between the substrate and the analyte. The SWCNT matrix film generated basically constant yield as a function of different SWCNT surface coverage. In the case of HOPG, the intensities of the parent-like ions were lower than the results of fullerene and SWCNT. The morphology and topography of the films support the proposed "colliding ball" model. This model offers a physical mechanism of SI enhancement by the fullerene matrix.

\section{Acknowledgments}

The authors acknowledge support for this research by ERDF OP R\&D, Project QUTE- Centrum excelentnosti kvantových technológií and APVV-0491-07.

\section{References}

1. Wei, J., Buriak, J.M., Siuzdak, G.: Desorption-Ionization Mass Spectrometry on Porous Silicon. Nature 399, 243-246 (1999)

2. Shen, Z.X., Siuzdak, G.: Porous Silicon as a Versatile Platform for Laser Desorption/Ionization Mass Spectrometry. Anal. Chem. 73, 612617 (2001)

3. Go, E.P., Apon, J.V., Luo, G., Saghatelian, A., Daniels, R.H., Sahi, V., Dubrow, R., Cravatt, B.F., Vertes, A., Siuzdak, G.: Desorption/ Ionization on Silicon Nanowires. Anal. Chem. 77, 1641-1646 (2005)

4. Xu, S., Li, Y., Zou, H., Qiu, J., Guo, Z., Guo, B.: Carbon Nanotubes as Assisted Matrix for Laser Desorption/Ionization Time-of-Flight Mass Spectrometry. Anal. Chem. 75, 6191-6195 (2003)

5. Wu, K.J., Odom, R.W.: Matrix Enhanced Secondary Ion Mass Spectrometry: A Method for Molecular Analysis of Solid Surfaces. Anal. Chem. 68, 873-882 (1996)

6. Adriaensen, L., Vangaever, F., Lenaerts, J., Gijbels, R.: MatrixEnhanced Secondary Ion Mass Spectrometry: The Influence of MALDI Matrices on Molecular Ion Yields of Thin Organic Films. Rapid Commun. Mass Spectrom. 19, 1017-1024 (2005)

7. Heeren, R.M.A., Kürker-Kaletaș, B., Taban, I.M., McAleese, L., McDonnell, L.A.: Quality of Surface: The Influence of Sample Preparation on MS-Based Biomolecular Tissue Imaging with MALDI-MS and (ME-) SIMS. Appl. Surf. Sci. 255, 1289-1297 (2008)

8. Lua, Y.-Y., Yang, L., Pew, C.A., Zhang, F., Fillmore, W.J.J., Bronson, R.T., Sathyapalan, A., Savage, P.B., Whittaker, J.D., Davis, R.C., Linford, M.R.: Polyelectrolytes as New Matrices for Secondary Ion Mass Spectrometry. J. Am. Soc. Mass Spectrom. 16, 1575-1582 (2005)

9. Huang, M.W., L.-Chei, H., Huang, J.P., Shiea, J.: Application of Organic Solvents as Matrixes to Detect Air-Sensitive and Less Polar Compounds Using Low-Temperature Secondary Ion Mass Spectrometry. Anal. Chem. 71, 2901-2907 (1999)

10. Delcorte, A., Bertrand, P.: Metal Salts for Molecular Ion Yield Enhancement in Organic Secondary Ion Mass Spectrometry: A Critical Assessment. Anal. Chem. 77, 2107-2115 (2005)

11. Delcorte, A., Bour, J., Aubriet, F., Muller, J.-F., Bertrand, P.: Sample Metallization for Performance Improvement in Desorption/ Ionization of Kilodalton Molecules: Quantitative Evaluation, Imaging Secondary ion MS, and Laser Ablation. Anal. Chem. 75, 68756885 (2003)

12. Heile, A., Lipinsky, D., Wehbe, N., Delcorte, A., Bertrand, P., Felten, A., Houssiau, L., Pireaux, J.-J., De Mondt, R., Van Vaeck, L., Arlinghaus, H.F.: Metal-Assisted SIMS and Cluster Ion Bombardment for Ion Yield Enhancement. Appl. Surf. Sci. 255, 941-943 (2008)

13. Delcorte, A., Bertrand, P.: Interest of Silver and Gold Metallization for Molecular SIMS and SIMS Imaging. Appl. Surf. Sci. 231/232, 250-255 (2004)

14. Michalak, L., Fisher, K., Alderdice, D., Willet, G.: $\mathrm{C}_{60}$-Assisted Laser Desorption/Ionization Mass Sspectrometry. Rapid Commun. Org. Mass Spectrom. 29, 512-515 (1994)

15. Tang, H.-W., Ng, K.-M., Lu, W., Che, C.-M.: Ion Desorption Efficiency and Internal Energy Transfer in Carbon-Based SurfaceAssisted Laser Desorption/Ionization Mass Spectrometry: Desorption Mechanism(s) and the Design of SALDI Substrates. Anal. Chem. 81, 4720-4722 (2009)

16. Shiea, J., Huang, J.-P., Teng, C.-F., Jeng, J., Wang, L.Y., Chiang, L.Y.: Use of a Water-Soluble Fullerene Derivative as Precipitating Reagent and Matrix-Assisted Laser Desorption/Ionization Matrix to Selectively Detect Charged Species in Aqueous Solutions. Anal. Chem. 75, 35873595 (2003)

17. Ugarov, M.V., Egan, T., Khabashesku, D.V., Schultz, J.A., Peng, H., Khabashesku, V.N., Furutani, H., Prather, K.S., Wang, H.-W.J., Jackson, S.N., Woods, A.S.: MALDI Matrices for Biomolecular Analysis Based on Functionalized Carbon Nanomaterials. Anal. Chem. 76, 6734-6742 (2004)

18. Pan, C., Xu, S., Zou, H., Guo, Z., Zhang, Y., Guo, B.: Using Oxidized Carbon Nanotubes as Matrix for Analysis of Small Molecules by MALDI-TOF MS. J. Am. Soc. Mass Spectrom. 16, 883-892 (2005)

19. Ren, S., Zhang, L., Cheng, Z., Guo, Y.: Immobilized Carbon Nanotubes as Matrix for MALDI-TOF-MS Analysis: Applications 
to Neutral Small Carbohydrates. J. Am. Soc. Mass Spectrom. 16, 333-339 (2005)

20. Delcorte, A., Garrison, B.J.: High Yield Events of Molecular Emission Induced by Kiloelectronvolt Particle Bombardment. J. Phys. Chem. B 104, 6785-6800 (2000)

21. Krantzman, K.D., Webb, R.P., Garrison, B.J.: Simulations of $\mathrm{C}_{60}$ Bombardment of Si, Sic, Diamond, and Graphite. Appl. Surf. Sci. 255, 837-840 (2008)
22. Campbell, E.E.D.: Fullerene Collision Reactions. Kluwer AP, New York (2004)

23. Dresselhaus, M.S., Dresselhaus, G., Eklund, P.: Science of Fullerenes and Carbon Nanotubes. Academic Press, San Diego (1996)

24. Dresselhaus, M.S., Dresselhaus, G., Avouris, P.: Carbon Nanotubes: Synthesis, Structure, Properties and Applications. Topics in Appl. Phys. 80, 247-272 (2001) 